\title{
Glucocorticoid-Induced Leucine Zipper: A Novel Anti-inflammatory Molecule
}

\section{OPEN ACCESS}

Edited by:

Arianna Carolina Rosa,

University of Turin, Italy

Reviewed by:

Mythily Srinivasan,

Indiana University Bloomington,

United States

Brendan E. Russ,

Monash University, Australia

Taylah Bennett,

Monash University, Melbourne, Australia, in collaboration with BR

*Correspondence:

Carlo Riccard

carlo.riccardi@unipg.it

Specialty section:

This article was submitted to

Experimental Pharmacology and Drug Discovery,

a section of the journal

Frontiers in Pharmacology

Received: 07 December 2018

Accepted: 12 March 2019

Published: 27 March 2019

Citation:

Bereshchenko O, Migliorati G, Bruscoli S and Riccardi C (2019) Glucocorticoid-Induced Leucine

Zipper: A Novel Anti-inflammatory Molecule. Front. Pharmacol. 10:308.

doi: 10.3389/fphar.2019.00308

\author{
Oxana Bereshchenko', Graziella Migliorati2 ${ }^{2}$ Stefano Bruscoli² and Carlo Riccardi* \\ 'Department of Surgery and Biomedical Sciences, University of Perugia, Perugia, Italy, ${ }^{2}$ Section of Pharmacology, \\ Department of Medicine, University of Perugia, Perugia, Italy
}

Glucocorticoids (GCs) are the most commonly used drugs for treatment of autoimmune and inflammatory diseases. Their efficacy is due to their ability to bind cytoplasmic receptors (glucocorticoid receptors, GR) and other cytoplasmic proteins, thus regulating gene expression. Although GCs are potent life-saving drugs, their therapeutic effects are transitory and chronic use of GCs is accompanied by serious side effects. Therefore, new drugs are needed to replace GCs. We have identified a gene, glucocorticoid-induced leucine zipper (GILZ or tsc22d3), that is rapidly and invariably induced by GCs. Human GILZ is a 135-amino acid protein that mediates many GC effects, including inhibition of the NF-кB and MAPK pathways. Similar to GCs, GILZ exerts anti-inflammatory activity in experimental disease models, including inflammatory bowel diseases and arthritis. While transgenic mice that overexpress GILZ are more resistant, GILZ knockout mice develop worse inflammatory diseases. Moreover, the anti-inflammatory effect of GCs is attenuated in GILZ-deficient mice. Importantly, in vivo delivery of recombinant GILZ protein cured colitis and facilitated resolution of lipopolysaccharide-induced inflammation without apparent toxic effects. A synthetic GILZ-derived peptide, corresponding to the GILZ region that interacts with NF-кB, was able to suppress experimental autoimmune encephalomyelitis. Collectively, these findings indicate that GILZ is an anti-inflammatory molecule that may serve as the basis for designing new therapeutic approaches to inflammatory diseases.

Keywords: glucocorticoid-induced leucine zipper, recombinant GILZ protein, GILZ, GILZ-derived peptide, anti-inflammatory molecules

\section{INTRODUCTION: GLUCOCORTICOID-INDUCED LEUCINE ZIPPER (GILZ) IS A GLUCOCORTICOID (GC)-INDUCIBLE GENE}

Glucocorticoids (GCs) are important hormones able to regulate homeostasis of virtually all organs and tissues of the human body. GCs act mainly as regulators of transcriptional activity of a large number of genes, but also by control of epigenetic mechanisms (Cain and Cidlowski, 2017; Zannas and Chrousos, 2017). GCs have anti-inflammatory and immunosuppressive activities that involve nearly all arms of the inflammatory response. Accordingly, GCs are widely used for therapy. However, although GCs are potent anti-inflammatory drugs, their clinical effects are transitory and chronic use of GCs is accompanied by serious side effects, such as hypertension, hyperglycemia, osteoporosis, mood disorders and Cushing's syndrome, that lead to discontinuation of therapy 
(Ayroldi et al., 2014). Therefore, new drugs that can substitute for GCs may provide a critical aid in suppressing inflammation. Most of the effects mediated by GCs, at physiological and pharmacological concentrations, are mediated by interactions with the glucocorticoid receptor (GR) (De Bosscher et al., 2003). GR is predominantly located in the cytoplasm as a multiprotein complex called the receptosome. After GC/GR interaction and consequent conformational changes, GR dissociates from the complex and translocates into the nucleus, where, as a dimer, it interacts with other transcription and coactivator factors and binds to specific DNA sequences, called glucocorticoid recognition elements (GREs). Almost all the effects of GCs, therapeutic and unwanted, are mediated by GR activity. Extensive efforts to separate beneficial from harmful gene activation by modulating GR activity have not yielded any success and GC toxicity is still a big issue in clinical practice. Since GC-induced effects are mostly due to the modulation of target gene expression, we initiated studies aimed at identifying proteins induced by GC treatment that mediate the anti-inflammatory effects, but not the side effects, of GCs. This is an emerging approach in drug design and is based on the development of peptide and mimetic drugs with decreased toxicity and enhanced specificity compared with conventional anti-inflammatory molecules.

Glucocorticoid-induced leucine zipper (GILZ, or tsc22d3) was discovered in our laboratory as a gene rapidly induced by dexamethasone (DEX), a synthetic GC (D'Adamio et al., 1997; Cannarile et al., 2001). In silico and mutational analyses of the GILZ promoter showed that at least three canonical GREs are present in the proximal region of the GILZ transcriptional start site (Asselin-Labat et al., 2004; Wang et al., 2004). More than 100 papers have been published showing that GILZ is one of the earliest transcriptional targets of GR, thus indicating that it is a good candidate as a downstream GC-induced effector molecule. GC signaling can also induce epigenetic modifications, such as changes in histone methylation and acetylation (Vockley et al., 2016). We have observed that GILZ binds and inhibits histone deacetylases in myoblasts, suggesting GILZ may also be involved in the effect of epigenetic changes induced by GCs (Bruscoli et al., 2010).

Of note, responsiveness of GC-inducible genes to activated GR may also be dependent on chromatin accessibility of the regulatory regions (enhancers and promoters) that may be influenced and affected by environmental stimuli. These epigenetic changes might influence expression of GC-induced genes, including GILZ (John et al., 2011; Stavreva et al., 2015). On the other hand, the secretion of GCs itself is a classic endocrine response to environmental exposures, including stress. Consequently, GILZ, induced by GCs, is produced in response to stressful events such as tissue damage, infection, anxiety or depression. Notably, it has been shown that major depressive disorder (MDD) patients with reduced GILZ expression in the hippocampus and amygdala showed a smaller hippocampal volume, suggesting the involvement of GILZ in stress-related disorders such as depression and anxiety (Frodl et al., 2012).

In this review, we describe experimental evidence suggesting that GILZ mediates the anti-inflammatory effects of GCs and that GILZ-based molecules have anti-inflammatory properties.

\section{GILZ MEDIATES THE ANTI-INFLAMMATORY ACTIVITY OF GCs}

In recent years, several laboratories including our own have produced a large amount of experimental evidence that supports the role of GILZ as a mediator of the antiinflammatory activity of GCs (Figure 1) (Ayroldi et al., 2014; Ronchetti et al., 2015). GILZ is involved in the modulation of the same signaling immune responses and inflammation-related pathways implicated in GCinduced anti-inflammatory and immunosuppressive activities (Figure 1), suggesting that targeting GILZ can constitute a new approach to the treatment of inflammatory/autoimmune diseases.

Glucocorticoid-induced leucine zipper was initially cloned in 1997 by comparing gene expression profiles of DEX-treated versus untreated murine $\mathrm{T}$ cells in differential display assays (D’Adamio et al., 1997). In this work, D'Adamio et al. (1997) showed that GILZ is involved in the regulation of $\mathrm{T}$ cell apoptosis. GILZ expression protects $\mathrm{T}$ cells from activated induced cell death through inhibition of Fas and FasL expression. The discovery of a human GILZ ortholog by Cannarile et al. (2001) indicated that GILZ is expressed and is induced by GCs in all peripheral blood cells, including $\mathrm{T}$ lymphocytes, monocytes and granulocytes. GILZ is also expressed in several other non-lymphoid tissues, and is ubiquitously upregulated by GCs (Cari et al., 2015). Much experimental evidence shows that GILZ interacts with several transcription factors and cellular signaling pathways (Table 1). Among those, GILZ physically interacts with essential factors in the control of inflammatory processes, including subunit p65 of nuclear factor kappa-light-chain-enhancer of activated B cells (NF-кB), c-Jun/c-Fos heterodimer (named Activator protein-1, AP-1), Raf-1, Ras, and CCAAT-enhancer-binding proteins (CEBPs) (Figure 2), as discussed in detail in the following sections, suggesting it has a remarkable central role in the control of inflammation.

\section{GILZ INTERACTION WITH NF- $\kappa B$ and MAPK/ERK PATHWAYS IN INFLAMMATION}

So far, the most relevant evidence of anti-inflammatory GILZ activities is related to interference with the NF- $\kappa \mathrm{B}$ and MitogenActivated Protein Kinase (MAPK)/extracellular signal-regulated kinases (ERK) pathways. NF- $\kappa \mathrm{B}$ is a transcription factor essential for the modulation of inflammatory and immune responses by controlling cytokine and chemokine production. Although NF- $\kappa$ B plays an essential, beneficial role in normal physiology, inappropriate regulation of NF- $\mathrm{B}$ activity has been implicated in the pathogenesis of diseases including rheumatoid arthritis (RA), inflammatory bowel diseases (IBDs), osteoarthritis, atherosclerosis, asthma, multiple sclerosis (MS), and cancer (Barnes and Karin, 1997; Dai et al., 2009; Lawrence, 2009; Ben-Neriah and Karin, 2011). 


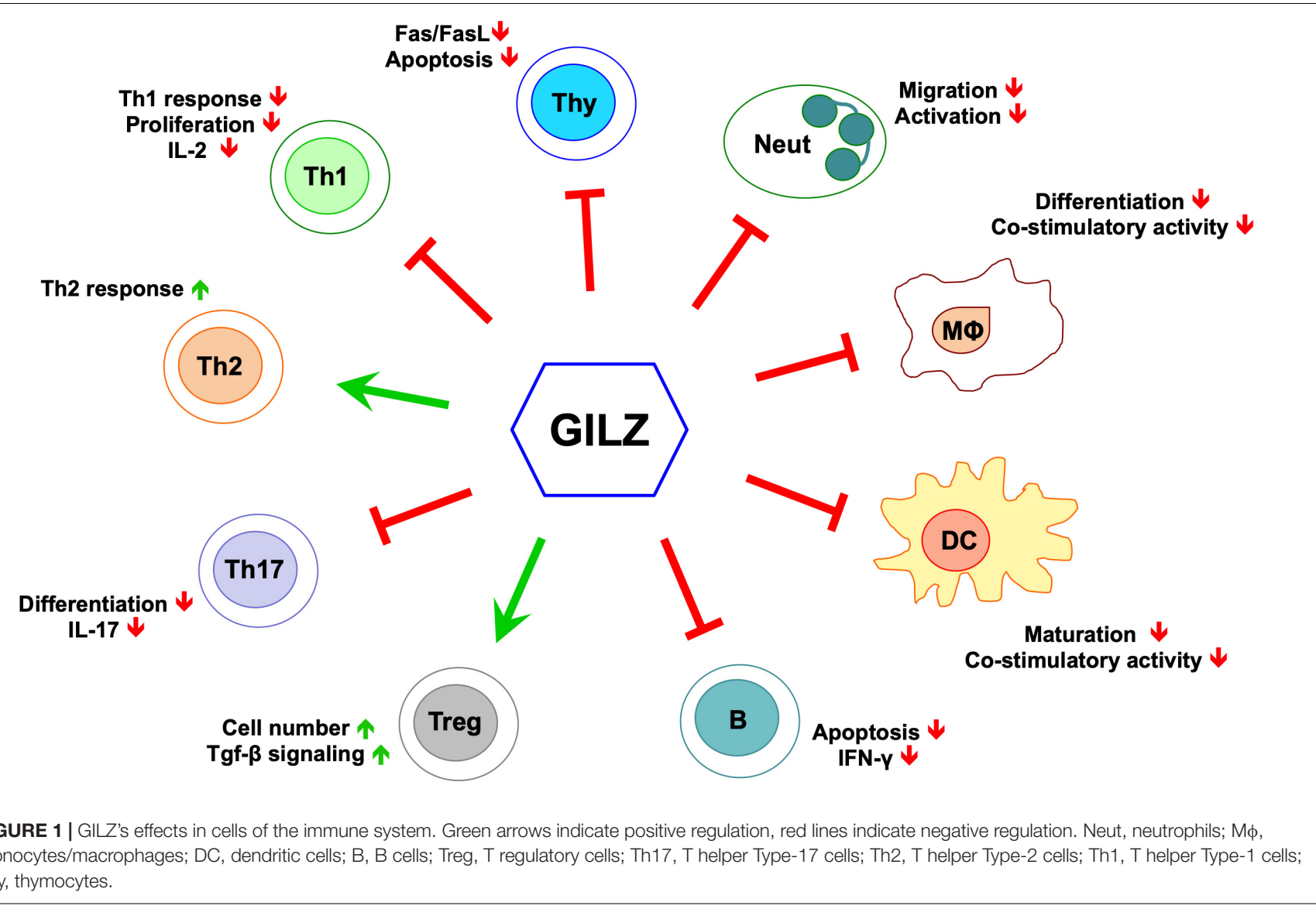

Interference with NF- $\mathrm{KB}$ transcriptional activity by GCs in monocytes, macrophages, neutrophils, dendritic cells (DCs) and $\mathrm{T}$ lymphocytes is very important for the inhibition of the inflammatory response. We proposed that GILZ up-regulation induced by GCs is another way in which GCs control the activation of NF- $\mathrm{\kappa B}$. Ayroldi et al. (2001) demonstrated that GILZ directly binds to NF-KB and inhibits its activation (Figure 2). They showed that GILZ overexpression blocks nuclear translocation of the NF- $\mathrm{kB} / \mathrm{p} 65$ subunit and it prevents the induction of pro-inflammatory NF- $\mathrm{KB}$ target genes in thymocytes, for example Interleukin-2 (IL-2) (Ayroldi et al., 2001; Di Marco et al., 2007). GILZ also mimics the effects of GCs on the differentiation of mature $\mathrm{T}$ cells, since GILZ overexpression in $T$ cells of GILZ transgenic mice induces downregulation of the $\mathrm{T}$ helper (Th)-1 response and upregulation of the Th-2 response (Cannarile et al., 2006). The shift toward a Th2 response correlates with inhibition of NF- $\mathrm{KB}$ nuclear translocation in $\mathrm{CD} 4+\mathrm{T}$ lymphocytes of intestinal lamina propria, and as a consequence, GILZ transgenic mice are less susceptible to Th1mediated colitis.

Subsequent experimental evidence, obtained using several in vitro and in vivo models, further supported the link between anti-inflammatory activity of GILZ and inhibition of NF- $\kappa$ B. Berrebi et al. (2003) showed that GC inhibition of macrophage activation is at least in part achieved by GILZmediated inhibition of NF-КB. GILZ is constitutively expressed in mouse and human macrophages and in human monocytes, and is upregulated by GCs (Cannarile et al., 2001; Berrebi et al., 2003). In the THP-1 macrophage cell line, GILZ binds to the p65/NF-KB subunit and inhibits the expression of costimulatory molecules CD80 and CD86 and the production of chemokines Regulated on Activation, Normal T Cell Expressed and Secreted (RANTES) and Macrophage inflammatory protein 1 (MIP-1), thus mimicking the effect of GC treatment on macrophages (Berrebi et al., 2003). It has also been shown that GILZ mediates immunosuppressive effects in DCs. Overexpression of GILZ led to a tolerogenic effect of DCs in mediating T-cell activation by interfering with the NF-kB pathway (Cohen et al., 2006; Benkhoucha et al., 2014).

Beside the reported functions on cells of the immune system, GILZ has also been implicated in regulation of epithelial and endothelial inflammatory response. Indeed, GILZ silencing in human alveolar macrophages led to increased pro-inflammatory response and NF- $\kappa$ B activity (Hoppstadter et al., 2012). GILZ is also expressed in epithelial cells and GILZ peptide in human airway epithelial BEAS-2B cell line suppressed NF- $\mathrm{BB}$ activation (Eddleston et al., 2007). Furthermore, GILZ is expressed in synovial endothelial cells in RA, where it has been suggested to modulate inflammatory leukocyte recruitment via NF- $\mathrm{B}$. GILZ overexpression inhibits endothelial cell adhesive function through decreased expression of E-selectin, Intercellular Adhesion Molecule 1 (ICAM-1), C-C Motif 
TABLE 1 | GILZ protein binding partners.

\begin{tabular}{llll}
\hline Gene name & Experimental system & Reference & PubMed ID \\
\hline JUN & In vitro binding assay & $\begin{array}{l}\text { Mittelstadt and } \\
\text { Ashwell, 2001 }\end{array}$ & 11397794 \\
FOS & In vitro binding assay & $\begin{array}{l}\text { Mittelstadt and } \\
\text { Ashwell, 2001 }\end{array}$ & 11397794 \\
NFKB2 (p52) & Affinity Capture-Western & Ayroldi et al., 2001 & 11468175 \\
NFKB1 (p65) & Affinity Capture-Western & Ayroldi et al., 2001 & 11468175 \\
RAF1 & Affinity Capture-Western & Ayroldi et al., 2002 & 12391160 \\
RAS & Affinity Capture-Western & Ayroldi et al., 2007 & 17492054 \\
SCNN1B & Affinity Capture-Western & Soundararajan et al., & 19380724 \\
& & 2009 & \\
MYOD1 & Affinity Capture-Western & Bruscoli et al., 2010 & 20124407 \\
HDAC2 & Affinity Capture-Western & Bruscoli et al., 2010 & 20124407 \\
HDAC1 & Affinity Capture-Western & Bruscoli et al., 2010 & 20124407 \\
UBE2l & In vitro binding assay & Delfino et al., 2011 & 20671745 \\
SUMO1 & In vitro binding assay & Delfino et al., 2011 & 20671745 \\
CASP8 & Affinity Capture-Western & Delfino et al., 2011 & 20671745 \\
SGK1 & Affinity Capture-Western & Soundararajan et al., & 20947508 \\
& & 2009 & \\
RAS & In vitro binding assay & Venanzi et al., 2014 & 24993177 \\
TP53 & In vitro binding assay & Ayroldi et al., 2014 & 25168242 \\
TP53 & Affinity Capture-Western & Ayroldi et al., 2014 & 25168242 \\
MDM2 & Affinity Capture-Western & Ayroldi et al., 2014 & 25168242 \\
PU.1 & Affinity Capture-Western & Ricci et al., 2017 & 28373208 \\
\hline & & &
\end{tabular}

Chemokine Ligand 2 (CCL2), C-X-C Motif Chemokine Ligand 8 (CXCL8), and Interleukin-6 (IL-6) and inhibition of Tumor Necrosis Factor (TNF)-stimulated leukocyte rolling, adhesion, and transmigration. Moreover, GILZ is downregulated in human umbilical vein endothelial cells by pro-inflammatory $\mathrm{TNF} \alpha$. Again, the anti-inflammatory effect of GILZ on endothelial cells involves the inhibition of NF- $\kappa \mathrm{B}$ (Hahn et al., 2014). Moreover, GILZ-mediated inhibition of NF- $\kappa \mathrm{B}$ nuclear translocation diminishes Cyclooxygenase 2 (COX-2) expression in bone marrow mesenchymal stem cells (MSCs) that were implicated in the pathogenesis and progression of RA (Chen and Tuan, 2008). GILZ expression was detected in the synovium of patients with RA and of mice with collagen-induced arthritis (CIA), and the severity of the disease was enhanced by GILZ silencing (Beaulieu et al., 2010; Ayroldi et al., 2014). The role of GILZ in the antiinflammatory effect of MSCs in RA involves modulation of Th17 response toward a regulatory phenotype (Luz-Crawford et al., 2015). Another study confirmed that GILZ acts as a negative regulator of IL-17 production by T cells (Jones et al., 2015). GILZ expression in salivary gland is decreased in a mouse model of Sjögren's syndrome, and GILZ overexpression inhibits production of IL-17 in salivary gland cells, suggesting that GILZ represents a potential target for diagnosis and treatment of Sjögren's syndrome (Qin et al., 2015). Biological functions of Th17 cells are usually associated with pro-inflammatory activity implicated in the pathogenesis of autoimmune diseases. Yosef et al. (2013) proposed a dynamic regulatory network that comprises distinct Th17 cells with pro-inflammatory or regulatory activities. They indicated GILZ as a potential marker of Th17 cells with a non-pathogenic status (Yosef et al., 2013).
It has also been shown that GILZ dampens the proinflammatory effects of TNF $\alpha$ in human adipocytes by inhibiting p65/NF- $\kappa$ B nuclear translocation (Lee et al., 2016).

We have also shown that GILZ plays an important role in the homeostasis of B-cells (Bruscoli et al., 2015, 2018). GILZ deficiency leads to increased B-cell survival through elevated NF- $\kappa \mathrm{B}$ activity and B-Cell CLL/Lymphoma 2 (Bcl-2) overexpression. B lymphocytosis observed in gilz deficient mice suggests that GILZ could be implicated in the pathogenesis of B-cell disorders such as hematological and/or autoimmune/inflammatory diseases. In this regard, a recent study reports a reduced GILZ expression in B cells of patients with systemic lupus erythematosus (SLE) (Jones et al., 2016).

The influence of GILZ on the expression of Bcl-2 family members is consistent with observations indicating that GILZ overexpression inhibits apoptosis in $\mathrm{T}$ lymphocytes through down-regulation of $\mathrm{Bcl}-2$ Interacting Mediator Of Cell Death (Bim), a pro-apoptotic member of the Bcl-2 family (Asselin-Labat et al., 2004). GILZ also inhibits apoptosis in cardiomyocytes, where the pro-survival isoform of Bcl$\mathrm{XL}$ protein is overexpressed when GILZ is overexpressed (Aguilar et al., 2014).

Interestingly, it has been shown that a GILZ-based peptide inhibits p65/NF-kB activation in lipopolysaccharide (LPS)stimulated THP-1 myeloid cell line (Srinivasan et al., 2014). More recently, another study showed that a peptide corresponding to the 98-134 amino acids of GILZ inhibits LPS-induced p65/NF-kB nuclear translocation in retinal Müller cells (Gu et al., 2018a).

Collectively, these findings suggest that GILZ or GILZbased peptides mimic the effect of GCs on NF- $\kappa$ B inhibition and GILZ expression is sufficient to achieve efficacious suppression of NF- $\kappa \mathrm{B}$-induced inflammation (Ngo et al., 2013; Ayroldi et al., 2014).

Many anti-inflammatory effects of GCs depend on cross-talk with the MAPK signaling pathway. We have shown that GILZ interacts with the MAPK pathway by binding to Raf- 1 and Ras (Figure 2) (Ayroldi et al., 2002, 2007). GILZ interaction with activated Ras results in inhibition of both the Phosphoinositide 3-kinase (PI3K)/Akt and ERK pathways, thus dampening cell growth (including tumor cell growth) and differentiation. Also, GILZ mediates DEX-induced inhibition of the MAPK/ERK pathway in human airway epithelial cells (Liu et al., 2013). Specific genetic ablation of GILZ in myeloid cells resulted in an increased susceptibility to LPS-induced macrophage activation through hyperactivity of the MAPK/ERK pathway (Hoppstadter et al., 2015). Furthermore, in cooperation with Annexin-1, another mediator of anti-inflammatory effects of GCs, GILZ inhibits LPS-induced macrophage activation by enhancement of the ERK and JUN N-Terminal Kinase (JNK) MAPK pathways (Yang et al., 2009), and neutrophil migration into inflamed tissues (Ricci et al., 2017). In addition, GILZ also inhibits neutrophil activation in a mouse model of $\mathrm{C}$. albicans infection (Ricci et al., 2019).

Inhibition of oncoprotein Ras affects both the MAPK and PI3k/Akt pathways that are involved in cell survival and activation of immune responses. Forkhead family (Fox) transcription factors are mediators in the PI3K/Akt pathway 


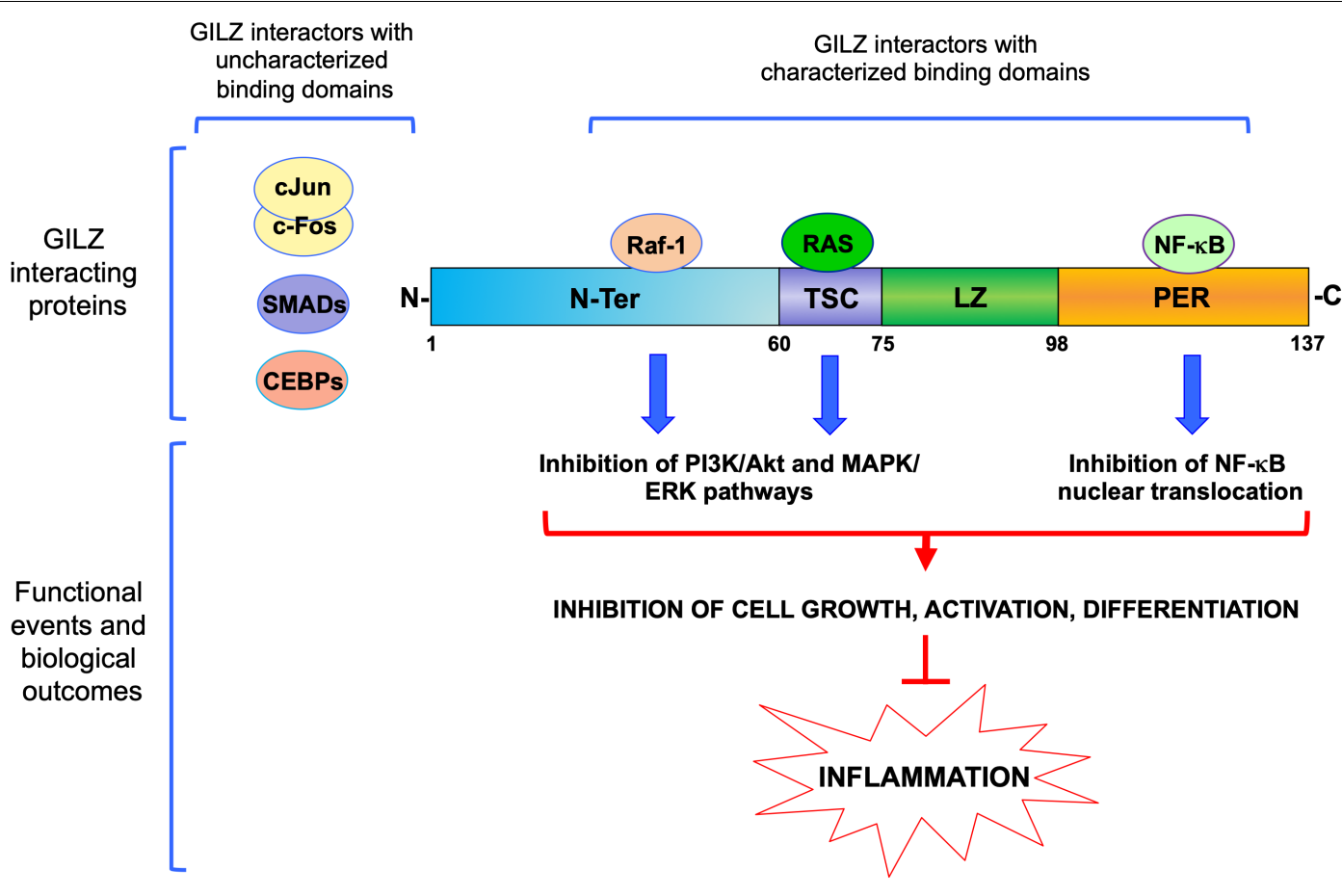

FIGURE 2 | Molecular interactions of GILZ in the control of inflammation. Schematic representation of GILZ's protein and its functional domains: N-Ter, N-terminal region (1-60 amino acids); TSC, TGF- $\beta$ stimulated clone box (61-75 amino acids); LZ, leucine zipper domain (76-97 amino acids); PER, proline (P) and glutamic acid (E) rich region (98-137 amino acids). Proteins with characterized region of interaction are represented above GILZ protein diagram or aside, when specific regions of interaction have not been characterized yet. Raf-1, Raf-1 Proto-Oncogene, Serine/Threonine Kinase; RAS, Ras Proto-Oncogene, GTPase; NF-кB, nuclear factor kappa-light-chain-enhancer of activated B cells; cJun/cFos, proto-Oncogene components of an heterodimer also named Activator protein-1, AP-1; SMADs, Mothers against decapentaplegic homolog proteins; CEBPs, CCAAT-enhancer-binding proteins.

in the homeostasis of immune cells, including $\mathrm{T}$ lymphocytes (Peng, 2008). Among these factors, FoxO3 is phosphorylated and inactivated by activated Akt. It has been shown that GILZ inhibits FoxO3 activity and is part of a PI3K/Akt-FoxO3 signaling pathway that controls cell proliferation (Latre de Late et al., 2010).

A recent interesting report by Hoppstadter et al. (2016) showed that GILZ is induced in macrophages by curcumin, a natural product with anti-inflammatory properties. Hoppstadter et al. (2016) showed that curcumin inhibits inflammatory activities of the NF- $\kappa$ B and MAPK/Erk pathways and subsequent TNF $\alpha$ production via GILZ (Hoppstadter et al., 2016).

\section{GILZ AND OTHER INFLAMMATORY SIGNALING PATHWAYS}

GILZ is able to inhibit the function of AP-1, a transcription factor pivotal for activation of immune cells during inflammation (Schonthaler et al., 2011). GILZ heterodimerizes with components of AP-1, c-Fos and c-jun (Mittelstadt and Ashwell, 2001), and GILZ overexpression inhibits production of IL-2, a cytokine that plays a central role in T-cell homeostasis and activation (Ayroldi et al., 2001; Mittelstadt and Ashwell, 2001; Di Marco et al., 2007). T-cell activation, also through IL-2 production, suppresses GILZ expression (D'Adamio et al., 1997;
Ayroldi et al., 2001; Asselin-Labat et al., 2004). This reciprocal inhibitory activity between T-cell activation and GILZ expression indicates that GILZ is important to maintain T-cell anergy, thus suggesting that modifications of GILZ expression and/or functions may have relevant effects in the control of inflammatory processes. Recently, we demonstrated that in GILZ-deficient mice, interferon- $\gamma$ (IFN $\gamma$ ) production by $B$ cells is increased, depending at least in part on enhanced AP-1 transcriptional activity. GILZ binds AP-1, inhibits its nuclear translocation and hence dampens AP-1-mediated transcriptional activation of IFN $\gamma$ promoter, thus regulating $B$ cell activities and the development of inflammatory diseases (Bruscoli et al., 2018).

As already mentioned, GILZ, like GCs, supports $\mathrm{T}$ cell differentiation toward the Th2 phenotype (Cannarile et al., 2006). An appropriate balance between inflammatory and antiinflammatory signals is dependent on the regulated activity of different $\mathrm{T}$ cell subpopulations and the ability of tissue to regulate the immune response (Hall et al., 2011). In particular, T regulatory (Treg) cells participate in modulation of inflammatory response and their deregulation has been shown to be involved in many inflammatory diseases (Rudensky, 2011). It is now clear that Treg induction is important for GCinduced therapeutic effects. GCs are able to synergize with Transforming growth factor beta (TGF- $\beta$ ) in regulating the expression of Foxp-3, a transcription factor essential for Treg 
generation (Rudensky, 2011; Bereshchenko et al., 2014). Foxp3 expression is in fact induced by TGF- $\beta$ pathway activation that is due to Mothers against decapentaplegic homolog (SMAD) protein phosphorylation; interaction of TGF- $\beta$ with specific receptors induces SMAD phosphorylation and consequently increases Foxp-3 expression. Bereshchenko et al. (2014) showed that GILZ is necessary for GC-mediated Treg cell induction. This effect is due to GILZ interaction with SMAD proteins, thus synergizing with the TGF- $\beta$ activity in enhancing FoxP3 expression (Bereshchenko et al., 2014). Notably, the therapeutic effect of GCs was lacking in a colitis model in GILZ knockout mice (Bereshchenko et al., 2014).

CEBPs are leucine zipper (LZ) transcription factors involved in diverse cellular responses including cell proliferation and differentiation, and also in inflammatory processes. There are several studies indicating a role for CEBP members in mediating GC effects (Roos and Nord, 2012). CEBPs are transcription factors that hetero-dimerize with many other proteins, including GC-target proteins such as MAPK phosphatase 1(MKP1)/dual specificity phosphatase 1 (DUSP1) (Kassel et al., 2001).

It has been shown that GILZ binds CEBP $\delta$ and inhibits peroxisome proliferator-activator receptor (PPAR)-gamma 2 expression, thus resulting in inhibition of differentiation adipogenesis in MSCs (Shi et al., 2003). Moreover, GILZ overexpression inhibits CEBP $\alpha$ expression and function, thus promoting osteogenesis in MSCs (Zhang et al., 2008). Although there is no direct evidence that GILZ is involved in the control of immunoregulatory molecules produced by MSCs, as GCs do, the aforementioned evidence supports the idea that GCs also affect CEBP activity through GILZ expression. Thus, more studies are warranted to understand the interplay between GILZ and CEPBs in mediating the anti-inflammatory effects of GCs in MSCs.

Serum/Glucocorticoid Regulated Kinase 1 (SGK1) is another important kinase that participates in the control of inflammation. Aberrant SGK1 expression is observed in a wide variety of diseases, including lung fibrosis, diabetic nephropathy, glomerulonephritis, obstructive nephropathy, liver cirrhosis, pancreatitis, and Crohn's disease (Artunc and Lang, 2014). Pharmacologic inhibition of SGK1 or suppression by small interfering RNA enhances pro-inflammatory cytokine production (including TNF, IL-12, and IL-6) in Toll Like Receptor (TLR)-activated monocytes. Lack of SGK1 results in increased phosphorylation of inhibitor of NF-kB kinase (IKK) and inhibitor of NF-kB Alpha (I $\mathrm{B} \alpha \alpha$ ), and consequent increased NF- $\mathrm{KB} / \mathrm{p} 65$ transcriptional activity in LPS-stimulated cells (Zhou et al., 2015). Interestingly, both SGK-1 and GILZ are up-regulated by aldosterone in the kidney and regulate the activity of Epithelial $\mathrm{Na}(+)$ Channel $(\mathrm{ENaC})$, a sodium transport channel (Bhalla et al., 2006). More recently, it has been reported that reduced SGK-1 and GILZ expression was concomitant with increased IL-6 expression in patients with MDD (Frodl et al., 2012). These indications suggest a possible cooperation of SGK-1 and GILZ in the control of inflammatory response pathways.

Many members of the nuclear receptor superfamily are involved in the modulation of inflammation. In particular, the PPARs, like GR, have anti-inflammatory properties because they reduce expression of several pro-inflammatory cytokines, chemokines and cell adhesion molecules. Three isoforms, PPAR- $\alpha$, PPAR- $\beta / \delta$, and PPAR- $\gamma$, form heterodimers with retinoic acid receptors. Deficiency of PPAR- $\alpha$ in mice impairs DEX-induced inhibition of NF- $\kappa B$, TNF- $\alpha$ production, cell migration, and COX-2 production. Moreover, PPAR- $\alpha$ contributes to the transcriptional up-regulation of GILZ by GCs (Cuzzocrea et al., 2008). GILZ over-expression inhibits PPAR- $\gamma 2$ expression and blocks adipocyte differentiation (Zhang et al., 2008). The interplay between PPARs, GCs and GILZ is still not well characterized, and further studies are warranted to clarify whether GILZ also affects PPAR functions in inflammatory processes.

Estrogen receptor (ER), another member of the nuclear receptor superfamily, regulates GILZ expression. Tynan et al. (2004) showed that estrogens modulate GILZ expression in MCF7 human breast cancer cells. Again, crosstalk between the GR and ER nuclear receptor members in inflammation might be therapeutically relevant and anti-inflammatory activity of GILZ may play a relevant role in this interplay (Cuzzocrea et al., 2007).

Together, these studies indicate that GILZ acts as a factor necessary for the maintenance of quiescence of immune cells. GILZ is downregulated upon activation of many cell types, including $\mathrm{T}$ and $\mathrm{B}$ lymphocytes, macrophages and DCs (D'Adamio et al., 1997; Lebson et al., 2011; Bruscoli et al., 2018; Hoppstadter et al., 2019), and is required for their proper activation, maturation and differentiation.

\section{GILZ STRUCTURE-BASED DESIGN OF PUTATIVE ANTI-INFLAMMATORY MOLECULES}

The mouse GILZ gene encodes a 137-amino acid (aa) LZ protein (D'Adamio et al., 1997). Human GILZ is a 135-aa protein, almost identical to its murine homologue (97\% identity) (Cannarile et al., 2001). GILZ is composed of three domains-a TGF- $\beta$-stimulated clone (TSC) box (aa positions 40-75), a central LZ domain (aa 76-97), and a C-terminal domain endowed with a proline and glutamic acid rich (PER) region (aa 98-137). We also characterized a transcriptional variant, named long (L)-GILZ, that shares with GILZ all the conserved domains including the TSC box, LZ, and PER region, but differs in the N-terminal domain, and is encoded by an upstream alternative exon 1 (Bruscoli et al., 2012). Unlike most LZ proteins, which are usually transcription factors, GILZ isoforms do not contain a basic-rich region, and so far there has been no formal demonstration of direct binding to DNA; GILZ is mostly located in the cytoplasm, although it may also be present in the nucleus (Shi et al., 2003).

Different domains have been identified as essential for direct protein-protein binding interactions with different molecules. For example, the TSC-box domain is important for binding with Ras, while Raf-1 binds the N-terminal portion of GILZ. Both Raf- 1 and Ras are activators of MAPK/ERK and PI3K/Akt pathways and GILZ expression negatively modulates both pathways (Ayroldi et al., 2002, 2007; Asselin-Labat et al., 2004; Venanzi et al., 2014). Interestingly, both c-Fos and c-Jun, proteins containing LZ domains and components of the transcription 
factor AP-1, are efficiently retained by the GILZ N-terminal portion (aa 1-60), which lacks the LZ domain necessary for homo-dimerization, indicating that the LZ domain is not necessary for GILZ to bind AP-1 components (Di Marco et al., 2007). This evidence opens an interesting question about the role of the LZ domain in GILZ. Usually, a LZ region is a protein-protein interaction motif in which there is a leucine every seventh residue in an $\alpha$-helix over a short stretch of protein, forming a stable coiled-coil structure. This motif is important for homo- and heterodimerization with a similar motif to promote specific DNA binding by a basic region adjacent to the LZ motif. The LZ model was originally proposed on the basis of the leucine distribution in $\mathrm{LZ}$ regions of proteins. It is now known to be common to over 40 proteins, including C/EBPs, Myc, Fos, Jun, and cAMP Responsive Element Binding Protein (CREB), involved in stress responses and the regulation of cell survival and proliferation (Jindrich and Degnan, 2016). LZ proteins can also associate with non-identical partners to form heterodimers composed of two different subunits, thus greatly expanding the repertoire of functions that these proteins can display. Although the LZ domain of GILZ is highly conserved, it has not yet been demonstrated to be necessary for heterodimerization with other $\mathrm{LZ}$ proteins. It is possible that the presence of the TSC-box domain, close to the LZ domain, limits the capacity of the GILZ LZ domain to bind other LZ family proteins. Interestingly, GILZ also binds to other LZ proteins such as $\mathrm{CEBP} \alpha, \mathrm{CEBP} \beta$ and $\mathrm{CEBP} \gamma$, but the region responsible for direct binding has not yet been identified (Shi et al., 2003; Pan et al., 2014).

Importantly, much experimental evidence reporting GILZ overexpression in mice, as well as in vivo delivery of recombinant GILZ protein, suggests that GILZ mimics some of the antiinflammatory effects of GCs, including inhibition of $\mathrm{T}$ cell activation/differentiation and macrophage activation (D'Adamio et al., 1997; Ayroldi et al., 2001; Berrebi et al., 2003; Cannarile et al., 2009; Pinheiro et al., 2013; Bereshchenko et al., 2014; Hoppstadter et al., 2015), thus supporting the idea that GILZ protein may serve as a basis for rational drug design to achieve similar anti-inflammatory effects to GCs but with reduced side effects.

The first demonstration that recombinant GILZ protein has anti-inflammatory effects was by Cannarile et al. (2009), when a transactivator of transcription (TAT)-GILZ fusion protein, injected subcutaneously in a mouse model of Dinitrobenzene sulfonic acid (DNBS)-induced colitis, was found efficacious in ameliorating signs of colon inflammation (Cannarile et al., 2009). It has also been demonstrated that hydrodynamic delivery of GILZ-expressing vectors containing the TAT-GILZ sequence was sufficient to prevent LPS-induced lethal inflammation (shock) (Pinheiro et al., 2013). In addition, Vago et al. (2015) have shown that TAT-GILZ fusion protein efficiently induces a pro-apoptotic program in vivo that promotes resolution of neutrophilic inflammation induced by LPS. Recently, it was shown that in vivo administration of TAT-GILZ fusion protein in a mouse model of acute kidney injury exerts protective effects by promoting neutrophil and $\mathrm{T}$ cell polarization toward an anti-inflammatory phenotype (Baban et al., 2018).
The use of GILZ-derived peptides containing the critical aa 120-123 necessary for GILZ interaction with NF- $\mathrm{B}$ (Di Marco et al., 2007) has been shown to effectively counteract neuroinflammation in a mouse model of MS (Srinivasan and Janardhanam, 2011). Recently, an in vivo injection of GILZbased peptide prevented light-induced photoreceptor apoptosis and protected the retina from degeneration in Sprague Dawley rats, thus demonstrating the therapeutic efficacy of GILZ-based peptide in degenerative retinal diseases (Gu et al., 2018b).

The inhibitory effect on neuroinflammation was also reported in a study where transgenic mice overexpressing GILZ showed reduced damage and inflammation upon spinal cord injury (SCI) compared with wild-type controls (Esposito et al., 2012). Interestingly, a different study showed that, in the same SCI model, genetic ablation of GILZ in T lymphocytes led to reduced lesions and inflammation, suggesting that GILZ is involved in inflammatory processes upon SCI (Mazzon et al., 2014). This apparent contradiction is probably because, apart from its direct immunomodulatory role on T cells (D'Adamio et al., 1997; Ronchetti et al., 2015) GILZ is also expressed in neurons and may have functions in cells involved in neuronal damage (Srinivasan and Lahiri, 2017). Further studies are warranted to define the role of GILZ in the central nervous system.

Finally, forced GILZ overexpression in endothelial cells inhibits their activation and production of chemoattractant molecules, through NF- $\kappa \mathrm{B}$ inhibition, thus reducing leukocyte recruitment to the site of inflammation (Cheng et al., 2013).

\section{CONCLUSION}

Data indicate that GILZ protein and GILZ-based molecules have therapeutic efficacy and are non-toxic to cells and mice. Therefore, novel drugs in the area of "inflammatory/autoimmune" diseases, including chronic, acute and lethal inflammation (shock), can be developed based on GILZ and its molecular interactions. Moreover, those drugs will be of interest for many inflammation-based degenerative diseases and possibly cancer. In the near future, we propose to identify novel anti-inflammatory drugs designed on the basis of the GILZ protein structure and described molecular interactions of GILZ that have been characterized in recent years. The efficacy, toxicity and molecular effects of these drugs will be evaluated in cellular systems and in established in vivo models of IBD and RA. A combination of experimental and computational techniques, together with a deep knowledge of the determinants of protein-protein and protein-ligand interactions, is necessary for the successful design of small compounds based on GILZ that will be efficacious for the treatment of inflammatory and autoimmune diseases.

\section{AUTHOR CONTRIBUTIONS}

$\mathrm{OB}, \mathrm{GM}$, and $\mathrm{CR}$ conceived the manuscript. $\mathrm{OB}$ and $\mathrm{SB}$ wrote the manuscript. SB designed the table and the figures. All authors approved the final version of the manuscript. 


\section{FUNDING}

This work was supported by the Italian Ministry of Education and Research, grants PRIN2015ZT9HXY to

\section{REFERENCES}

Aguilar, D., Strom, J., and Chen, Q. M. (2014). Glucocorticoid induced leucine zipper inhibits apoptosis of cardiomyocytes by doxorubicin. Toxicol. Appl. Pharmacol. 276, 55-62. doi: 10.1016/j.taap.2014.01.013

Artunc, F., and Lang, F. (2014). Mineralocorticoid and SGK1-sensitive inflammation and tissue fibrosis. Nephron. Physiol. 128, 35-39. doi: $10.1159 / 000368267$

Asselin-Labat, M. L., David, M., Biola-Vidamment, A., Lecoeuche, D., Zennaro, M. C., Bertoglio, J., et al. (2004). GILZ, a new target for the transcription factor FoxO3, protects T lymphocytes from interleukin-2 withdrawal-induced apoptosis. Blood 104, 215-223. doi: 10.1182/blood-2003-12-4295

Ayroldi, E., Macchiarulo, A., and Riccardi, C. (2014). Targeting glucocorticoid side effects: selective glucocorticoid receptor modulator or glucocorticoid-induced leucine zipper? A perspective. FASEB J. 28, 5055-5070. doi: 10.1096/fj.14254755

Ayroldi, E., Migliorati, G., Bruscoli, S., Marchetti, C., Zollo, O., Cannarile, L., et al. (2001). Modulation of T-cell activation by the glucocorticoid-induced leucine zipper factor via inhibition of nuclear factor kappaB. Blood 98, 743-753. doi: 10.1182/blood.V98.3.743

Ayroldi, E., Zollo, O., Bastianelli, A., Marchetti, C., Agostini, M., Di Virgilio, R., et al. (2007). GILZ mediates the antiproliferative activity of glucocorticoids by negative regulation of Ras signaling. J. Clin. Invest. 117, 1605-1615. doi: 10.1172/JCI30724

Ayroldi, E., Zollo, O., Macchiarulo, A., Di Marco, B., Marchetti, C., and Riccardi, C. (2002). Glucocorticoid-induced leucine zipper inhibits the Raf-extracellular signal-regulated kinase pathway by binding to Raf-1. Mol. Cell. Biol. 22, 79297941. doi: 10.1128/MCB.22.22.7929-7941.2002

Baban, B., Marchetti, C., Khodadadi, H., Malik, A., Emami, G., Lin, P. C., et al. (2018). Glucocorticoid-induced leucine zipper promotes neutrophil and T-cell polarization with protective effects in acute kidney injury. J. Pharmacol. Exp. Ther. 367, 483-493. doi: 10.1124/jpet.118.251371

Barnes, P. J., and Karin, M. (1997). Nuclear factor-kappaB: a pivotal transcription factor in chronic inflammatory diseases. N. Engl. J. Med. 336, 1066-1071. doi: 10.1056/NEJM199704103361506

Beaulieu, E., Ngo, D., Santos, L., Yang, Y. H., Smith, M., Jorgensen, C., et al. (2010). Glucocorticoid-induced leucine zipper is an endogenous antiinflammatory mediator in arthritis. Arthritis Rheum. 62, 2651-2661. doi: 10.1002/art. 27566

Benkhoucha, M., Molnarfi, N., Dunand-Sauthier, I., Merkler, D., Schneiter, G., Bruscoli, S., et al. (2014). Hepatocyte growth factor limits autoimmune neuroinflammation via glucocorticoid-induced leucine zipper expression in dendritic cells. J. Immunol. 193, 2743-2752. doi: 10.4049/jimmunol.1302338

Ben-Neriah, Y., and Karin, M. (2011). Inflammation meets cancer, with NF-kappaB as the matchmaker. Nat. Immunol. 12, 715-723. doi: 10.1038/ni.2060

Bereshchenko, O., Coppo, M., Bruscoli, S., Biagioli, M., Cimino, M., Frammartino, T., et al. (2014). GILZ promotes production of peripherally induced Treg cells and mediates the crosstalk between glucocorticoids and TGF-beta signaling. Cell Rep. 7, 464-475. doi: 10.1016/j.celrep.2014. 03.004

Berrebi, D., Bruscoli, S., Cohen, N., Foussat, A., Migliorati, G., Bouchet-Delbos, L., et al. (2003). Synthesis of glucocorticoid-induced leucine zipper (GILZ) by macrophages: an anti-inflammatory and immunosuppressive mechanism shared by glucocorticoids and IL-10. Blood 101, 729-738. doi: 10.1182/blood2002-02-0538

Bhalla, V., Soundararajan, R., Pao, A. C., Li, H., and Pearce, D. (2006). Disinhibitory pathways for control of sodium transport: regulation of ENaC by SGK1 and GILZ. Am. J. Physiol. Renal Physiol. 291, F714-F721. doi: 10.1152/ajprenal. 00061.2006

Bruscoli, S., Biagioli, M., Sorcini, D., Frammartino, T., Cimino, M., Sportoletti, P., et al. (2015). Lack of glucocorticoid-induced leucine zipper (GILZ) deregulates
$\mathrm{CR}$ and RBFR13BN6Y to OB, by the Fondazione Cassa Risparmio Perugia Grant 2018.0410 021 and by University Funding for Basic Research - Assignment 2018 to GM.

B-cell survival and results in B-cell lymphocytosis in mice. Blood 126, 17901801. doi: 10.1182/blood-2015-03-631580

Bruscoli, S., Donato, V., Velardi, E., Di Sante, M., Migliorati, G., Donato, R., et al. (2010). Glucocorticoid-induced leucine zipper (GILZ) and long GILZ inhibit myogenic differentiation and mediate anti-myogenic effects of glucocorticoids. J. Biol. Chem. 285, 10385-10396. doi: 10.1074/jbc.M109.070136

Bruscoli, S., Sorcini, D., Flamini, S., Gagliardi, A., Adamo, F., Ronchetti, S., et al. (2018). Glucocorticoid-Induced Leucine Zipper Inhibits Interferon-Gamma Production in B Cells and Suppresses Colitis in Mice. Front. Immunol. 9:120. doi: 10.3389/fimmu.2018.01720

Bruscoli, S., Velardi, E., Di Sante, M., Bereshchenko, O., Venanzi, A., Coppo, M., et al. (2012). Long glucocorticoid-induced leucine zipper (L-GILZ) protein interacts with ras protein pathway and contributes to spermatogenesis control. J. Biol. Chem. 287, 1242-1251. doi: 10.1074/jbc.M111.316372

Cain, D. W., and Cidlowski, J. A. (2017). Immune regulation by glucocorticoids. Nat. Rev. Immunol. 17, 233-247. doi: 10.1038/nri.2017.1

Cannarile, L., Cuzzocrea, S., Santucci, L., Agostini, M., Mazzon, E., Esposito, E., et al. (2009). Glucocorticoid-induced leucine zipper is protective in Th1mediated models of colitis. Gastroenterology 136, 530-541. doi: 10.1053/j.gastro. 2008.09.024

Cannarile, L., Fallarino, F., Agostini, M., Cuzzocrea, S., Mazzon, E., Vacca, C., et al. (2006). Increased GILZ expression in transgenic mice up-regulates Th-2 lymphokines. Blood 107, 1039-1047. doi: 10.1182/blood-2005-05-2183

Cannarile, L., Zollo, O., D’Adamio, F., Ayroldi, E., Marchetti, C., Tabilio, A., et al. (2001). Cloning, chromosomal assignment and tissue distribution of human GILZ, a glucocorticoid hormone-induced gene. Cell Death. Differ. 8, 201-203. doi: $10.1038 /$ sj.cdd. 4400798

Cari, L., Ricci, E., Gentili, M., Petrillo, M. G., Ayroldi, E., Ronchetti, S., et al. (2015). A focused Real Time PCR strategy to determine GILZ expression in mouse tissues. Results Immunol. 5, 37-42. doi: 10.1016/j.rinim.2015.10.003

Chen, F. H., and Tuan, R. S. (2008). Mesenchymal stem cells in arthritic diseases. Arthritis Res. Ther. 10:223. doi: 10.1186/ar2514

Cheng, Q., Fan, H., Ngo, D., Beaulieu, E., Leung, P., Lo, C. Y., et al. (2013). GILZ overexpression inhibits endothelial cell adhesive function through regulation of NF-kappaB and MAPK activity. J. Immunol. 191, 424-433. doi: 10.4049/ jimmunol.1202662

Cohen, N., Mouly, E., Hamdi, H., Maillot, M. C., Pallardy, M., Godot, V., et al. (2006). GILZ expression in human dendritic cells redirects their maturation and prevents antigen-specific T lymphocyte response. Blood 107, 2037-2044. doi: 10.1182/blood-2005-07-2760

Cuzzocrea, S., Bruscoli, S., Crisafulli, C., Mazzon, E., Agostini, M., Muia, C., et al. (2007). Estrogen receptor antagonist fulvestrant (ICI 182,780) inhibits the anti-inflammatory effect of glucocorticoids. Mol. Pharmacol. 71, 132-144. doi: 10.1124/mol.106.029629

Cuzzocrea, S., Bruscoli, S., Mazzon, E., Crisafulli, C., Donato, V., Di Paola, R., et al. (2008). Peroxisome proliferator-activated receptor-alpha contributes to the anti-inflammatory activity of glucocorticoids. Mol. Pharmacol. 73, 323-337. doi: 10.1124/mol.107.041475

D’Adamio, F., Zollo, O., Moraca, R., Ayroldi, E., Bruscoli, S., Bartoli, A., et al. (1997). A new dexamethasone-induced gene of the leucine zipper family protects $\mathrm{T}$ lymphocytes from TCR/CD3-activated cell death. Immunity 7 , 803-812. doi: 10.1016/S1074-7613(00)80398-2

Dai, Y., Lawrence, T. S., and Xu, L. (2009). Overcoming cancer therapy resistance by targeting inhibitors of apoptosis proteins and nuclear factor-kappa B. Am. J. Transl. Res. 1, 1-15.

De Bosscher, K., Vanden Berghe, W., and Haegeman, G. (2003). The interplay between the glucocorticoid receptor and nuclear factor-kappaB or activator protein-1: molecular mechanisms for gene repression. Endocr. Rev. 24, 488-522. doi: 10.1210/er.2002-0006

Delfino, D. V., Spinicelli, S., Pozzesi, N., Pierangeli, S., Velardi, E., Bruscoli, S., et al. (2011). Glucocorticoid-induced activation of caspase- 8 protects the 
glucocorticoid-induced protein Gilz from proteasomal degradation and induces its binding to SUMO-1 in murine thymocytes. Cell Death Differ. 18, 183-190. doi: $10.1038 / \mathrm{cdd} .2010 .86$

Di Marco, B., Massetti, M., Bruscoli, S., Macchiarulo, A., Di Virgilio, R., Velardi, E., et al. (2007). Glucocorticoid-induced leucine zipper (GILZ)/NFkappaB interaction: role of GILZ homo-dimerization and C-terminal domain. Nucleic Acids Res. 35, 517-528. doi: 10.1093/nar/gkl1080

Eddleston, J., Herschbach, J., Wagelie-Steffen, A. L., Christiansen, S. C., and Zuraw, B. L. (2007). The anti-inflammatory effect of glucocorticoids is mediated by glucocorticoid-induced leucine zipper in epithelial cells. J. Allergy Clin. Immunol. 119, 115-122. doi: 10.1016/j.jaci.2006.08.027

Esposito, E., Bruscoli, S., Mazzon, E., Paterniti, I., Coppo, M., Velardi, E., et al. (2012). Glucocorticoid-induced leucine zipper (GILZ) over-expression in T lymphocytes inhibits inflammation and tissue damage in spinal cord injury. Neurotherapeutics 9, 210-225. doi: 10.1007/s13311-011-0084-7

Frodl, T., Carballedo, A., Hughes, M. M., Saleh, K., Fagan, A., Skokauskas, N., et al. (2012). Reduced expression of glucocorticoid-inducible genes GILZ and SGK-1: high IL-6 levels are associated with reduced hippocampal volumes in major depressive disorder. Transl. Psychiatry 2:e88. doi: 10.1038/tp. 2012.14

Gu, R., Ding, X., Tang, W., Lei, B., Jiang, C., and Xu, G. (2018a). A synthesized glucocorticoid- induced leucine zipper peptide inhibits retinal muller cell gliosis. Front. Pharmacol. 9:331. doi: 10.3389/fphar.2018.00331

Gu, R., Tang, W., Lei, B., Jiang, C., Song, F., and Xu, G. (2018b). Synthesized glucocorticoid-induced leucine zipper peptide inhibits photoreceptor apoptosis and protects retinal function in light-induced retinal degeneration model. Clin. Exp. Ophthalmol. doi: 10.1111/ceo.13452 [Epub ahead of print].

Hahn, R. T., Hoppstadter, J., Hirschfelder, K., Hachenthal, N., Diesel, B., Kessler, S. M., et al. (2014). Downregulation of the glucocorticoid-induced leucine zipper (GILZ) promotes vascular inflammation. Atherosclerosis 234, 391-400. doi: 10.1016/j.atherosclerosis.2014.03.028

Hall, B. M., Verma, N. D., Tran, G. T., and Hodgkinson, S. J. (2011). Distinct regulatory $\mathrm{CD} 4+\mathrm{T}$ cell subsets; differences between naive and antigen specific T regulatory cells. Curr. Opin. Immunol. 23, 641-647. doi: 10.1016/j.coi.2011. 07.012

Hoppstadter, J., Diesel, B., Eifler, L. K., Schmid, T., Brune, B., and Kiemer, A. K. (2012). Glucocorticoid-induced leucine zipper is downregulated in human alveolar macrophages upon Toll-like receptor activation. Eur. J. Immunol. 42, 1282-1293. doi: 10.1002/eji.201142081

Hoppstadter, J., Diesel, B., Linnenberger, R., Hachenthal, N., Flamini, S., Minet, M., et al. (2019). Amplified Host Defense by Toll-Like Receptor-Mediated Downregulation of the Glucocorticoid-Induced Leucine Zipper (GILZ) in Macrophages. Front. Immunol. 9:3111. doi: 10.3389/fimmu.2018.03111

Hoppstadter, J., Hachenthal, N., Valbuena-Perez, J. V., Lampe, S., Astanina, K., Kunze, M. M., et al. (2016). Induction of glucocorticoid-induced leucine zipper (GILZ) contributes to anti-inflammatory effects of the natural product curcumin in macrophages. J. Biol. Chem. 291, 22949-22960. doi: 10.1074/jbc. M116.733253

Hoppstadter, J., Kessler, S. M., Bruscoli, S., Huwer, H., Riccardi, C., and Kiemer, A. K. (2015). Glucocorticoid-induced leucine zipper: a critical factor in macrophage endotoxin tolerance. J. Immunol. 194, 6057-6067. doi: 10.4049/ jimmunol.1403207

Jindrich, K., and Degnan, B. M. (2016). The diversification of the basic leucine zipper family in eukaryotes correlates with the evolution of multicellularity. BMC Evol. Biol. 16:28. doi: 10.1186/s12862-016-0598-z

John, S., Sabo, P. J., Thurman, R. E., Sung, M. H., Biddie, S. C., Johnson, T. A., et al. (2011). Chromatin accessibility pre-determines glucocorticoid receptor binding patterns. Nat. Genet. 43, 264-268. doi: 10.1038/ng.759

Jones, S. A., Perera, D. N., Fan, H., Russ, B. E., Harris, J., and Morand, E. F. (2015). GILZ regulates Th17 responses and restrains IL-17-mediated skin inflammation. J. Autoimmun. 61, 73-80. doi: 10.1016/j.jaut.2015.05.010

Jones, S. A., Toh, A. E., Odobasic, D., Oudin, M. A., Cheng, Q., Lee, J. P., et al. (2016). Glucocorticoid-induced leucine zipper (GILZ) inhibits B cell activation in systemic lupus erythematosus. Ann. Rheum. Dis. 75, 739-747. doi: 10.1136/ annrheumdis-2015-207744

Kassel, O., Sancono, A., Kratzschmar, J., Kreft, B., Stassen, M., and Cato, A. C. (2001). Glucocorticoids inhibit MAP kinase via increased expression and decreased degradation of MKP-1. EMBO J. 20, 7108-7116. doi: 10.1093/emboj/ 20.24.7108

Latre de Late, P., Pepin, A., Assaf-Vandecasteele, H., Espinasse, C., Nicolas, V., Asselin-Labat, M. L., et al. (2010). Glucocorticoid-induced leucine zipper (GILZ) promotes the nuclear exclusion of FOXO3 in a Crm1-dependent manner. J. Biol. Chem. 285, 5594-5605. doi: 10.1074/jbc.M109.068346

Lawrence, T. (2009). The nuclear factor NF-kappaB pathway in inflammation. Cold Spring Harb. Perspect. Biol. 1:a001651. doi: 10.1101/cshperspect.a001651

Lebson, L., Wang, T., Jiang, Q., and Whartenby, K. A. (2011). Induction of the glucocorticoid-induced leucine zipper gene limits the efficacy of dendritic cell vaccines. Cancer Gene Ther. 18, 563-570. doi: 10.1038/cgt.2011.23

Lee, M. J., Yang, R. Z., Karastergiou, K., Smith, S. R., Chang, J. R., Gong, D. W., et al. (2016). Low expression of the GILZ may contribute to adipose inflammation and altered adipokine production in human obesity. J. Lipid Res. 57, 1256-1263. doi: 10.1194/jlr.M067728

Liu, J., Zhang, M., Niu, C., Luo, Z., Dai, J., Wang, L., et al. (2013). Dexamethasone inhibits repair of human airway epithelial cells mediated by glucocorticoidinduced leucine zipper (GILZ). PLoS One 8:e60705. doi: 10.1371/journal.pone. 0060705

Luz-Crawford, P., Tejedor, G., Mausset-Bonnefont, A. L., Beaulieu, E., Morand, E. F., Jorgensen, C., et al. (2015). Glucocorticoid-induced leucine zipper governs the therapeutic potential of mesenchymal stem cells by inducing a switch from pathogenic to regulatory Th17 cells in a mouse model of collagen-induced arthritis. Arthritis Rheumatol. 67, 1514-1524. doi: 10.1002/art.39069

Mazzon, E., Bruscoli, S., Galuppo, M., Biagioli, M., Sorcini, D., Bereshchenko, O., et al. (2014). Glucocorticoid-induced leucine zipper (GILZ) controls inflammation and tissue damage after spinal cord injury. CNS Neurosci. Ther. 20, 973-981. doi: $10.1111 / \mathrm{cns} .12315$

Mittelstadt, P. R., and Ashwell, J. D. (2001). Inhibition of AP-1 by the glucocorticoid-inducible protein GILZ. J. Biol. Chem. 276, 29603-29610. doi: 10.1074/jbc.M101522200

Ngo, D., Beaulieu, E., Gu, R., Leaney, A., Santos, L., Fan, H., et al. (2013). Divergent effects of endogenous and exogenous glucocorticoid-induced leucine zipper in animal models of inflammation and arthritis. Arthritis Rheum. 65, 1203-1212. doi: 10.1002/art.37858

Pan, G., Cao, J., Yang, N., Ding, K., Fan, C., Xiong, W. C., et al. (2014). Role of glucocorticoid-induced leucine zipper (GILZ) in bone acquisition. J. Biol. Chem. 289, 19373-19382. doi: 10.1074/jbc.M113.535237

Peng, S. L. (2008). Foxo in the immune system. Oncogene 27, 2337-2344. doi: 10.1038/onc.2008.26

Pinheiro, I., Dejager, L., Petta, I., Vandevyver, S., Puimege, L., Mahieu, T., et al. (2013). LPS resistance of SPRET/Ei mice is mediated by Gilz, encoded by the Tsc22d3 gene on the X chromosome. EMBO Mol. Med. 5, 456-470. doi: 10.1002/emmm.201201683

Qin, X., Liu, J. Y., Abdelsayed, R., Shi, X., Yu, J. C., Mozaffari, M. S., et al. (2015). The status of glucocorticoid-induced leucine zipper protein in the salivary glands in Sjogren's syndrome: predictive and prognostic potentials. EPMA J. 7:3. doi: 10.1186/s13167-016-0052-8

Ricci, E., Ronchetti, S., Gabrielli, E., Pericolini, E., Gentili, M., Roselletti, E., et al. (2019). GILZ restrains neutrophil activation by inhibiting the MAPK pathway. J. Leukoc. Biol. 105, 187-194. doi: 10.1002/JLB.3AB0718-255R

Ricci, E., Ronchetti, S., Pericolini, E., Gabrielli, E., Cari, L., Gentili, M., et al. (2017). Role of the glucocorticoid-induced leucine zipper gene in dexamethasone-induced inhibition of mouse neutrophil migration via control of annexin A1 expression. FASEB J. 31, 3054-3065. doi: 10.1096/fj.201601 $315 \mathrm{R}$

Ronchetti, S., Migliorati, G., and Riccardi, C. (2015). GILZ as a Mediator of the Anti-Inflammatory Effects of Glucocorticoids. Front. Endocrinol. 6:170. doi: 10.3389/fendo.2015.00170

Roos, A. B., and Nord, M. (2012). The emerging role of C/EBPs in glucocorticoid signaling: lessons from the lung. J. Endocrinol. 212, 291-305. doi: 10.1530/JOE11-0369

Rudensky, A. Y. (2011). Regulatory T cells and Foxp3. Immunol. Rev. 241, 260-268. doi: 10.1111/j.1600-065X.2011.01018.x

Schonthaler, H. B., Guinea-Viniegra, J., and Wagner, E. F. (2011). Targeting inflammation by modulating the Jun/AP-1 pathway. Ann. Rheum. Dis. 70(Suppl. 1), i109-i112. doi: 10.1136/ard.2010.140533 
Shi, X., Shi, W., Li, Q., Song, B., Wan, M., Bai, S., et al. (2003). A glucocorticoidinduced leucine-zipper protein, GILZ, inhibits adipogenesis of mesenchymal cells. EMBO Rep. 4, 374-380. doi: 10.1038/sj.embor.embor805

Soundararajan, R., Melters, D., Shih, I.-C., Wang, J., and Pearce, D. (2009). Epithelial sodium channel regulated by differential composition of a signaling complex. Proc. Natl. Acad. Sci. U.S.A. 106, 7804-7809. doi: 10.1073/pnas. 0809892106

Srinivasan, M., Blackburn, C., and Lahiri, D. K. (2014). Functional characterization of a competitive peptide antagonist of p65 in human macrophage-like cells suggests therapeutic potential for chronic inflammation. Drug Des. Devel. Ther. 8, 2409-2421. doi: 10.2147/DDDT.S59722

Srinivasan, M., and Janardhanam, S. (2011). Novel p65 binding glucocorticoidinduced leucine zipper peptide suppresses experimental autoimmune encephalomyelitis. J. Biol. Chem. 286, 44799-44810. doi: 10.1074/jbc.M111. 279257

Srinivasan, M., and Lahiri, D. K. (2017). Glucocorticoid-Induced Leucine Zipper in Central Nervous System Health and Disease. Mol. Neurobiol. 54, 8063-8070. doi: 10.1007/s12035-016-0277-5

Stavreva, D. A., Coulon, A., Baek, S., Sung, M. H., John, S., Stixova, L., et al. (2015). Dynamics of chromatin accessibility and long-range interactions in response to glucocorticoid pulsing. Genome Res. 25, 845-857. doi: 10.1101/gr.184168.114

Tynan, S. H., Lundeen, S. G., and Allan, G. F. (2004). Cell type-specific bidirectional regulation of the glucocorticoid-induced leucine zipper (GILZ) gene by estrogen. J. Steroid Biochem. Mol. Biol. 91, 225-239. doi: 10.1016/j.jsbmb.2004. 05.002

Vago, J. P., Tavares, L. P., Garcia, C. C., Lima, K. M., Perucci, L. O., Vieira, E. L., et al. (2015). The role and effects of glucocorticoid-induced leucine zipper in the context of inflammation resolution. J. Immunol. 194, 4940-4950. doi: 10.4049/jimmunol.1401722

Venanzi, A., Di Sante, M., Bruscoli, S., Biagioli, M., Sorcini, D., Cimino, M., et al. (2014). Recombinant long-glucocorticoid-induced leucine zipper (LGILZ) protein restores the control of proliferation in gilz $\mathrm{KO}$ spermatogonia. Eur. J. Pharm. Sci. 63, 22-28. doi: 10.1016/j.ejps.2014.06.013

Vockley, C. M., D’Ippolito, A. M., McDowell, I. C., Majoros, W. H., Safi, A., Song, L., et al. (2016). Direct GR Binding Sites Potentiate Clusters of TF Binding across the Human Genome. Cell 166:1269-1281.e19. doi: 10.1016/j.cell.2016. 07.049

Wang, J. C., Derynck, M. K., Nonaka, D. F., Khodabakhsh, D. B., Haqq, C., and Yamamoto, K. R. (2004). Chromatin immunoprecipitation (ChIP) scanning identifies primary glucocorticoid receptor target genes. Proc. Natl. Acad. Sci. U.S.A. 101, 15603-15608. doi: 10.1073/pnas.0407008101

Yang, Y. H., Aeberli, D., Dacumos, A., Xue, J. R., and Morand, E. F. (2009). Annexin-1 regulates macrophage IL-6 and TNF via glucocorticoid-induced leucine zipper. J. Immunol. 183, 1435-1445. doi: 10.4049/jimmunol.0804000

Yosef, N., Shalek, A. K., Gaublomme, J. T., Jin, H., Lee, Y., Awasthi, A., et al. (2013). Dynamic regulatory network controlling TH17 cell differentiation. Nature 496, 461-468. doi: 10.1038/nature11981

Zannas, A. S., and Chrousos, G. P. (2017). Epigenetic programming by stress and glucocorticoids along the human lifespan. Mol. Psychiatry 22, 640-646. doi: $10.1038 / \mathrm{mp} .2017 .35$

Zhang, W., Yang, N., and Shi, X. M. (2008). Regulation of mesenchymal stem cell osteogenic differentiation by glucocorticoid-induced leucine zipper (GILZ). J. Biol. Chem. 283, 4723-4729. doi: 10.1074/jbc.M70414 7200

Zhou, H., Gao, S., Duan, X., Liang, S., Scott, D. A., Lamont, R. J., et al. (2015). Inhibition of serum- and glucocorticoid-inducible kinase 1 enhances TLRmediated inflammation and promotes endotoxin-driven organ failure. FASEB J. 29, 3737-3749. doi: 10.1096/fj.15-270462

Conflict of Interest Statement: The authors declare that the research was conducted in the absence of any commercial or financial relationships that could be construed as a potential conflict of interest.

Copyright (C) 2019 Bereshchenko, Migliorati, Bruscoli and Riccardi. This is an openaccess article distributed under the terms of the Creative Commons Attribution License (CC BY). The use, distribution or reproduction in other forums is permitted, provided the original author(s) and the copyright owner(s) are credited and that the original publication in this journal is cited, in accordance with accepted academic practice. No use, distribution or reproduction is permitted which does not comply with these terms. 